\title{
ON CLUSTER ALGEBRAS FROM UNPUNCTURED SURFACES WITH ONE MARKED POINT
}

\author{
ILKE CANAKCI, KYUNGYONG LEE, AND RALF SCHIFFLER \\ (Communicated by Harm Derksen)
}

\begin{abstract}
We extend the construction of canonical bases for cluster algebras from unpunctured surfaces to the case where the number of marked points on the boundary is one. We show that the cluster algebra is equal to the upper cluster algebra in this case.
\end{abstract}

\section{INTRODUCTION}

Cluster algebras were introduced in [FZ1, and further developed in [FZ2, BFZ, FZ4, motivated by combinatorial aspects of canonical bases in Lie theory [L1, L2]. A cluster algebra is a subalgebra of a field of rational functions in several variables, and it is given by constructing a distinguished set of generators, the cluster variables. These cluster variables are constructed recursively and their computation is rather complicated in general. By construction, the cluster variables are rational functions, but Fomin and Zelevinsky showed in FZ1 that they are Laurent polynomials with integer coefficients. Moreover, these coefficients are known to be non-negative [LS].

An important class of cluster algebras is given by cluster algebras of surface type GSV, FG1, FG2, FST, FT. From a classification point of view, this class is very important, since it has been shown in FeShTu that almost all (skew-symmetric) mutation finite cluster algebras are of surface type. For generalizations to the skew-symmetrizable case see FeShTu2, FeShTu3]. The closely related surface skein algebras were studied in $\mathrm{M}, \mathrm{T}$.

If $\mathcal{A}$ is a cluster algebra of surface type, then there exists a surface with (possibly empty) boundary and marked points such that the cluster variables of $\mathcal{A}$ are in bijection with certain isotopy classes of curves, called arcs, in the surface. Marked points in the interior of the surface are called punctures, and the surface is unpunctured if all marked points lie on the boundary. Moreover, the relations between the cluster variables are given by the crossing patterns of the arcs in the surface. In [MSW], building on earlier work [S2, ST, S3, MS], the authors gave a combinatorial

Received by the editors August 23, 2014.

2010 Mathematics Subject Classification. Primary 13F60.

Key words and phrases. Cluster algebra, upper cluster algebra, unpunctured surface, basis.

The first author was supported by EPSRC grant number EP/K026364/1, UK and the University of Leicester.

The second author was supported by Wayne State University, the Korea Institute for Advanced Study, AMS Centennial Fellowship and NSA grant H98230-14-1-0323.

The third author was supported by NSF grants DMS-1254567, DMS-1101377 and by the University of Connecticut. 
formula for the cluster variables in cluster algebras of surface type. In the sequel MSW2, the formula was the key ingredient for the construction of two bases for the cluster algebra, in the case where the surface has no punctures and has at least two marked points.

1.1. Bases. Our main result, Theorem 2 shows that the basis construction of [MSW2 also applies to surfaces without punctures and with exactly one marked point. The proof of this result consists in showing that the Laurent polynomials associated to the essential loops in the surface are elements of the cluster algebra. This is shown by exhibiting certain identities in the cluster algebra, that allow us to write the Laurent polynomials in question as polynomials in cluster variables. The main ingredients for the proof of these identities are the snake graph calculus developed in [CS, CS2, CS3], and the skein relations proved in [MW].

1.2. Upper cluster algebras. As an application, we study the relationship between cluster algebras and upper cluster algebras. To define a cluster algebra $\mathcal{A}$, one needs to specify an initial seed $\Sigma=(\mathbf{x}, B)$ consisting of a cluster $\mathbf{x}$ and an exchange matrix $B$. In $\mathrm{BFZ}$, the authors introduced the concept of the upper cluster algebra $\mathcal{U}$ associated to the seed $\Sigma$. Recall that $\mathcal{U}$ consists of all elements of the ambient field $\mathbb{Q}\left(x_{1}, \ldots, x_{n}\right)$ which are Laurent polynomials over $\mathbb{Z}$ in the cluster variables from any seed in $\mathcal{A}$. It follows directly from the Laurent phenomenon that the upper cluster algebra contains the cluster algebra as a subalgebra. However, the question of whether the cluster algebra is equal to the upper cluster algebra is subtle and does not have a uniform answer. Already in BFZ it is shown that $\mathcal{A}=\mathcal{U}$ for all acyclic types and, on the other hand, $\mathcal{A} \neq \mathcal{U}$ for the rank 3 case given by the once-punctured torus. Muller introduced the notion of locally acyclic cluster algebras in [M2] and showed that $\mathcal{A}=\mathcal{U}$ for all locally acyclic types in [M3]. It is shown in MSp that cluster algebras of Grassmannians are locally acyclic, hence $\mathcal{A}=\mathcal{U}$ in this case. Goodearl and Yakimov announced that $\mathcal{A}=\mathcal{U}$ for double Bruhat cells GY].

For cluster algebras of finite mutation type, the following results are known:

(1) $\mathcal{A}=\mathcal{U}$ in the following types:

- surfaces with non-empty boundary and at least two marked points. This has been shown in MSW2 for unpunctured surfaces, and in M2 if at least two marked points are on the boundary. The case where only one point is on the boundary can be reduced to the case with two marked points on the boundary using the Louise property of $\mathrm{MSp}$; see Proposition 12 .

- the exceptional types $\mathbb{E}_{6}, \mathbb{E}_{7}, \mathbb{E}_{8}, \widetilde{\mathbb{E}}_{6}, \widetilde{\mathbb{E}}_{7}, \widetilde{\mathbb{E}}_{8}$, by [BFZ], since these types are acyclic.

- the exceptional types $\widetilde{\mathbb{E}}_{6}, \widetilde{\mathbb{E}}_{7}, \widetilde{\mathbb{E}}_{8}$ and $\mathbb{X}_{6}$, by $[\underline{\mathrm{M}}$, since they are locally acyclic, or by MSp, since they satisfy the Louise property.

(2) $\mathcal{A} \neq \mathcal{U}$ in the following types:

- surfaces without boundary and with exactly one puncture. This has been shown for the torus in [BFZ] and for higher genus in [Lad].

- the exceptional type $\mathbb{X}_{7}$, M4 .

As an application of Theorem 2, we prove in Theorem 5 that $\mathcal{A}=\mathcal{U}$ for surfaces without punctures and exactly one marked point. 
This leaves the question open only for surfaces without boundary and at least two punctures.

Summarizing, we have the following result.

Theorem 1. For all unpunctured surfaces, the cluster algebra is equal to the upper cluster algebra.

1.3. Maximal green sequences. Let $Q$ be a quiver and let $\bar{Q}$ be the quiver obtained from $Q$ by adding a vertex $i^{\prime}$ and an arrow $i \rightarrow i^{\prime}$, for each vertex $i$ of $Q$. The original vertices of $Q$ are called unfrozen (or mutable) and the new vertices are called frozen. A green sequence is a sequence of mutations starting at $\bar{Q}$ such that at each step the mutation is performed at an unfrozen vertex $i$, for which there is no arrow $j^{\prime} \rightarrow i$, from a frozen vertex $j^{\prime}$. A green sequence is maximal if it produces a quiver which does not contain such a vertex $i$.

Maximal green sequences have been introduced in $[\mathrm{K}$. They are related to quantum dilogarithm identities, DT invariants and BPS states; see BDP] and the references therein.

The question of whether or not there exists a quiver in the mutation class of $Q$ which admits a maximal green sequence seems related to the question of whether or not $\mathcal{A}(Q)=\mathcal{U}(Q)$. Indeed, given a surface $(S, M)$ which is either a surface with boundary, or a sphere with at least four punctures, or a torus with at least two punctures, there exists a triangulation $T$ whose quiver $Q_{T}$ admits a maximal green sequence. This has been shown in [ACCERV]; see also BDP for the sphere. Moreover, for all the exceptional types except for $\mathbb{X}_{7}$ there exists a quiver that admits a maximal green sequence [ACCERV], whereas the mutation class $\mathbb{X}_{7}$ does not contain a quiver that admits a maximal green sequence $\mathrm{Se}$.

On the other hand, it is shown in BDP, Proposition 8.1] that if $Q$ admits a non-degenerate potential such that the Jacobian algebra is infinite dimensional, then $Q$ does not admit maximal green sequences. Combining this result with GLS, Proposition 9.13], we see that quivers from closed surfaces with exactly one puncture do not admit maximal green sequences. The question seems to be open for closed surfaces of genus at least 2 and with at least two punctures.

Beyond finite mutation type, explicit maximal green sequences are announced by Yakimov for double Bruhat cells.

In all the known cases, maximal green sequences exist if and only if the cluster algebra is equal to the upper cluster algebra. Our Theorem 5 confirms this observation for unpunctured surfaces with exactly one marked point.

\section{MAIN RESULT}

Let $\mathcal{A}=\mathcal{A}(S, M)$ be a cluster algebra of an unpunctured surface $S$ with marked points $M$ whose coefficient system is such that the initial exchange matrix has maximal rank. The main result of [MSW2] is the construction of two bases for $\mathcal{A}$ under the assumption that the number of marked points is at least two. These are the bangles basis $\mathcal{B}_{0}$ and the bracelet basis $\mathcal{B}$. The assumption that the number of marked points is at least two was used in MSW2 only to show that all elements of $\mathcal{B}_{0}$ and $\mathcal{B}$ are actually inside the cluster algebra, and not only in the upper cluster algebra. The proof of the spanning property and of linear independence does not rely on the number of marked points.

The following theorem removes the assumption on the number of marked points. 
Theorem 2. Let $\mathcal{A}$ be the cluster algebra of an unpunctured surface with exactly one marked point and with arbitrary coefficients. Then both $\mathcal{B}$ and $\mathcal{B}_{0}$ are bases of the cluster algebra $\mathcal{A}$.

For the proof of this theorem, we need the following two lemmas. An essential loop in the surface $(S, M)$ is a closed curve in $S$ which is disjoint from the boundary of $S$, which is not contractible and does not have any self-crossings. Since $|M|=$ 1, the surface $S$ has exactly one boundary component. In MSW2, the authors associate to every essential loop a Laurent polynomial given as a sum over perfect matchings of the corresponding band graph.

Lemma 3. The Laurent polynomial $L$ of the essential loop around the boundary is in the cluster algebra $\mathcal{A}$.

Lemma 4. The Laurent polynomial $x_{\zeta}$ of every essential loop $\zeta$ is in the cluster algebra $\mathcal{A}$.

Of course the second lemma implies the first, but the first is needed in the proof of the second. We prefer stating them separately, because their proofs use different techniques. The proof of Lemma 3 is given in Section 3 and the proof of Lemma 4 in Section 4.

Proof of Theorem 2. Let $T$ be a triangulation of the surface and let $B_{T}$ be the associated exchange matrix. Since there are no punctures and the unique boundary component has an odd number of marked points (namely one), it follows from [FST, Theorem 14.3] that the rank of the matrix $B_{T}$ is maximal for every coefficient system. Therefore the results of [MSW2] show that both $\mathcal{B}_{0}$ and $\mathcal{B}$ are linearly independent and that every element of $\mathcal{A}$ is a linear combination of elements of $\mathcal{B}_{0}$ and also a linear combination of elements of $\mathcal{B}$.

It remains to show that $\mathcal{B}_{0}$ and $\mathcal{B}$ are subsets of $\mathcal{A}$.

Lemma 4 implies that every element of $\mathcal{B}_{0}$ is in $\mathcal{A}$. Moreover the Laurent polynomials associated to the bracelets in $\mathcal{B}$ can be written as Chebyshev polynomials in the Laurent polynomials associated to the essential loops in $\mathcal{B}_{0}$; see MSW2, Proposition 4.2]. Thus $\mathcal{B}$ is also a subset of $\mathcal{A}$.

The following result follows directly from Theorem 2

Theorem 5. Let $\mathcal{A}$ be the cluster algebra of an unpunctured surface with exactly one marked point and with trivial coefficients, and let $\mathcal{U}$ be its upper cluster algebra. Then

$$
\mathcal{A}=\mathcal{U}
$$

Proof. It follows from [FG1, Theorem 12.3(ii)] that $\mathcal{B}_{0}$ is a basis of $\mathcal{U}$. Theorem 2 implies that $\mathcal{B}_{0} \subset \mathcal{A}$, and thus $\mathcal{U} \subset \mathcal{A}$. The other inclusion always holds.

\section{Proof of Lemma 3}

We prove the lemma first for genus 1 , then genus 2 and then for higher genus. Throughout the section, we denote by $\mathcal{A}$ • the cluster algebra with principal coefficients in the initial seed corresponding to our triangulation $T$ and by $\hat{\mathcal{A}}$ a cluster algebra with arbitrary coefficient system and initial seed corresponding to $T$. More precisely, let $\mathbb{P}=\operatorname{Trop}\left(y_{1}, \ldots, y_{n}\right)$ be the tropical semifield and $\mathbf{y}=\left(y_{1}, \ldots, y_{n}\right)$ the initial coefficient tuple consisting of the generators of $\mathbb{P}$. Then $\mathcal{A}_{\bullet}$ is the cluster 
algebra with initial seed $\left(\mathbf{x}_{T}, \mathbf{y}, B_{T}\right)$. On the other hand, let $\hat{\mathbb{P}}$ be any semifield and $\hat{\mathbf{y}}=\left(\hat{y}_{1}, \ldots, \hat{y}_{n}\right)$ any coefficient tuple, thus $\hat{y}_{i} \in \hat{\mathbb{P}}$. Then $\hat{\mathcal{A}}$ is the cluster algebra with initial seed $\left(\mathbf{x}_{T}, \hat{\mathbf{y}}, B_{T}\right)$.

Recall that every arc or loop in $(S, M)$ corresponds to a snake or band graph and to a Laurent polynomial given by perfect matchings of the snake or band graph. A band graph is obtained from a snake graph by identifying an edge in the first tile with an edge in the last tile. In the figures, this identification is represented by marking the vertices of the identified edges with bullets. For precise definitions, we refer to [MSW2]. For the snake graph calculus used in this section, see CS2].

3.1. Genus 1. Let $(S, M)$ be a surface of genus 1 with one boundary component and one marked point. This case is known in the literature as 'the dreaded torus', because, among cluster algebras of surface type, it was the smallest example where the question of whether the cluster algebra is equal to the upper cluster algebra was open; see [MM, Remark 7.2.3]. Fix the triangulation $T$ shown on the left in Figure 1, and let $\mathbf{x}_{T}=\left(x_{1}, \ldots, x_{4}\right)$ be the corresponding cluster in $\mathcal{A}_{\bullet}$.
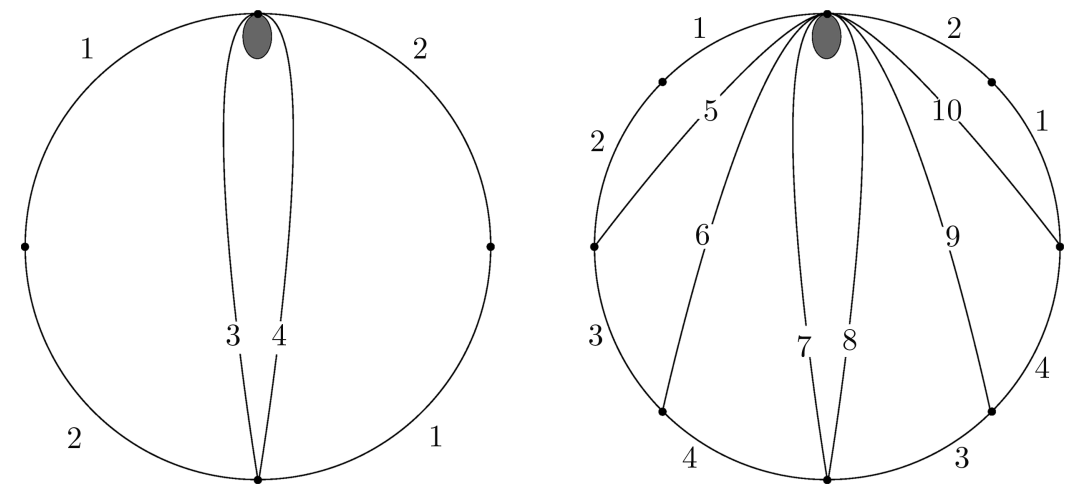

Figure 1. A triangulation of $(S, M)$ in genus 1 (left) and a triangulation in genus 2 (right).

Let $L$ be the Laurent polynomial associated to the loop around the boundary. Its band graph is the first graph in the second row in Figure 2

Lemma 6. $L \in \mathcal{A}_{\bullet}$.

Proof. Let $V_{1}$ be the cluster variable given by the arc that starts at the marked point in the direction between the arc 3 and the boundary and then crosses the $\operatorname{arcs} 4,2,1,4$ in order and ends at the same marked point as it started from in the direction between the arc 3 and the boundary. Let $V_{2}$ be the cluster variable given by the symmetric arc, that is, the arc that starts at the marked point in the direction between the arc 4 and the boundary and then crosses the $\operatorname{arcs} 3,1,2,3$ in order and ends at the same marked point as it started from in the direction between the arc 4 and the boundary. The snake graphs of these two cluster variables are the first two snake graphs in the top row of Figure 2. The first two equations in that figure use snake graph calculus of [CS] and CS2 to compute the product $V_{1} V_{2}$ showing that

$$
V_{1} V_{2}=L+y_{3}\left(y_{4} X_{1}+x_{3}\right)\left(X_{1}+y_{1} y_{2} y_{3} x_{4}\right)
$$




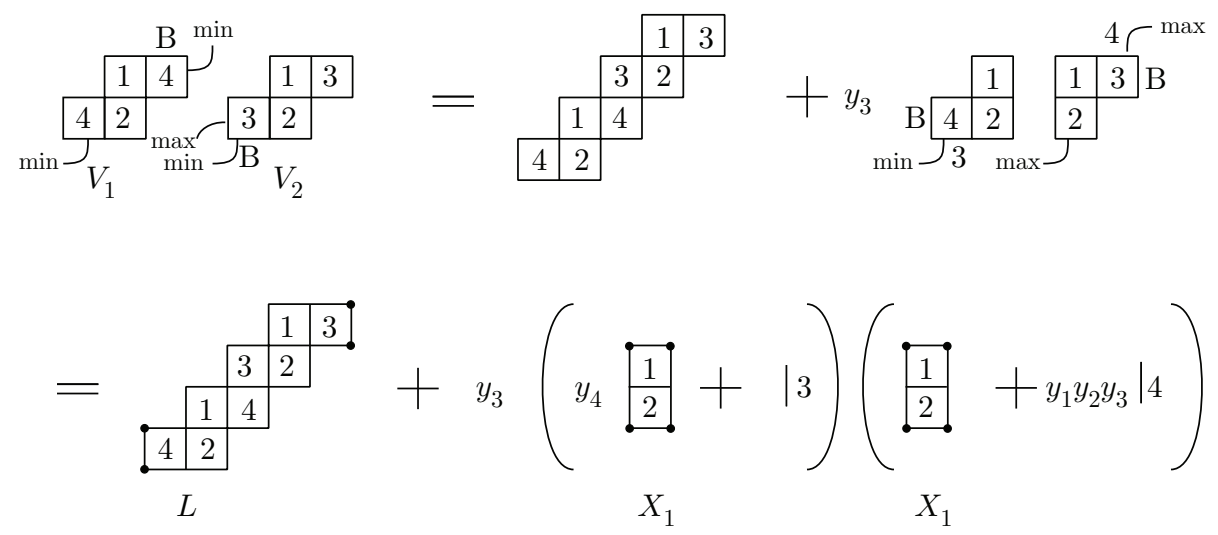

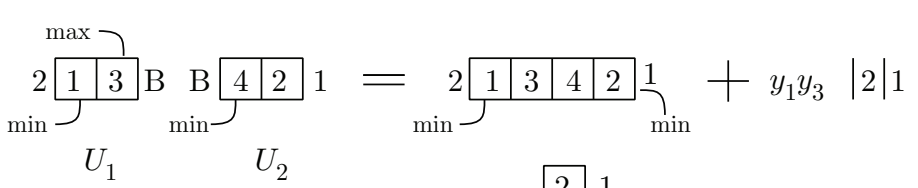

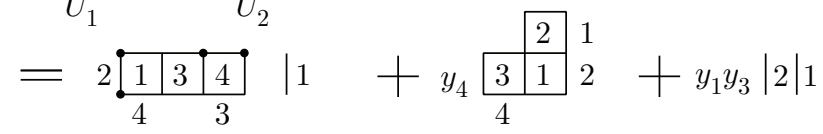

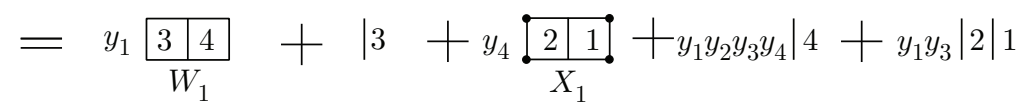

Figure 2. Snake graph calculus with principal coefficients. The edge labels 'min', 'max' refer to minimal and maximal matchings, which is needed only to determine the $y$-coefficients. The edge label $B$ refers to the boundary.

where $X_{1}$ is the Laurent polynomial defined by the corresponding band graph in the figure. The first equation is the resolution of the grafting operation [CS, section 2.5, case 2] and the second equation is the resolution of three self-grafting operations [CS2, section 3.4]. The graphs of $L$ and $X_{1}$ are band graphs.

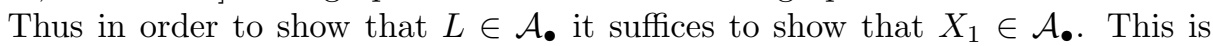
done in the second computation in Figure 2, where we compute the product $U_{1} U_{2}$ of the two cluster variables given by the arcs that cross 1,3 and 4,2 respectively, showing that

$$
U_{1} U_{2}=y_{1} W_{1}+x_{3}+y_{4} X_{1}+y_{1} y_{2} y_{3} y_{4} x_{4}+y_{1} y_{3} x_{1} x_{2},
$$

where $W_{1}$ is the Laurent polynomial defined by the corresponding snake graph in the figure. One can show that $U_{1}, U_{2}$ are cluster variables by checking that the associated arcs in the surface do not have a self-crossing, or by using CS2 to show that their associated snake graphs do not have any self-crossing overlap. The first equation in this computation is the resolution of a grafting, the second equation is a self-grafting CS2, section 3.3] and the third is a grafting of a band graph with a single edge [CS3 producing $y_{1} W_{1}+x_{3}$, as well as a self-grafting to obtain 
$y_{4} X_{1}+y_{1} y_{2} y_{3} y_{4} x_{4}$. Note that $W_{1}$ is a cluster variable corresponding to the arc crossing 3 and 4 . This shows that $y_{4} X_{1} \in \mathcal{A}_{\bullet}$. Since $y_{4}$ is an element of the group $\mathbb{P}$, its inverse $y_{4}^{-1}$ is also in $\mathbb{P}$, and therefore $X_{1} \in \mathcal{A}_{\bullet}$, and thus $L \in \mathcal{A}_{\bullet}$.

Lemma 7. $L \in \hat{\mathcal{A}}$.

Proof. Using Fomin-Zelevinsky's separation of addition formula [FZ4, Theorem 3.7] we see that the elements of $\hat{\mathcal{A}}$ can be computed from the elements in $\mathcal{A}$ by replacing the principal coefficients $y_{i}$ by the corresponding $\hat{y}_{i}$ and by dividing by the $F$ polynomial evaluated over $\hat{\mathbb{P}}$ in $\hat{y}_{i}$. For example,

$$
\hat{U}_{1}=\frac{U_{1}\left(x_{1}, \ldots, x_{n} ; \hat{y}_{1}, \ldots, \hat{y}_{n}\right)}{\hat{F}_{U_{1}}},
$$

where $\hat{F}_{U_{1}}=\left.F_{U_{1}}\right|_{\hat{\mathbb{P}}}\left(\hat{y}_{1}, \ldots, \hat{y}_{n}\right) \in \hat{\mathbb{P}} \subset \hat{\mathcal{A}}$.

Evaluating the expressions in equations (11) and (2) in $\hat{\mathbf{y}}$ clearly preserves the identities. Then multiplying with the $F$-polynomials we get

$$
\hat{V}_{1} \hat{V}_{2} \hat{F}_{V_{1}} \hat{F}_{V_{2}}=\hat{L} \hat{F}_{L}+\hat{y}_{3}\left(\hat{y}_{4} \hat{X}_{1} \hat{F}_{X_{1}}+x_{3}\right)\left(\hat{X}_{1} \hat{F}_{X_{1}}+\hat{y}_{1} \hat{y}_{2} \hat{y}_{3} x_{4}\right),
$$

$$
\hat{U}_{1} \hat{U}_{2} \hat{F}_{U_{1}} \hat{F}_{U_{2}}=\hat{y}_{1} \hat{W}_{1} \hat{F}_{W_{1}}+x_{3}+\hat{y}_{4} \hat{X}_{1} \hat{F}_{X_{1}}+\hat{y}_{1} \hat{y}_{2} \hat{y}_{3} \hat{y}_{4} x_{4}+\hat{y}_{1} \hat{y}_{3} x_{1} x_{2} .
$$

From equation (4) we see that $\hat{y}_{4} \hat{X}_{1} \hat{F}_{X_{1}} \in \hat{\mathcal{A}}$, and since $\hat{y}_{4} \in \hat{\mathbb{P}}$, and thus $\hat{y}_{4}^{-1} \in \hat{\mathbb{P}}$, this also implies that $\hat{X}_{1} \hat{F}_{X_{1}} \in \hat{\mathcal{A}}$. Now equation (3) implies that $\hat{L} \hat{F}_{L} \in \hat{\mathcal{A}}$, and, since $\hat{F}_{L} \in \hat{\mathbb{P}}$, this shows that $\hat{L} \in \hat{\mathcal{A}}$.

3.2. Genus 2. Let $(S, M)$ be a surface of genus 2 with one boundary component and one marked point. Fix the triangulation $T$ shown on the right in Figure 1 , and let $\mathbf{x}_{T}=\left(x_{1}, \ldots, x_{10}\right)$ be the corresponding cluster in $\mathcal{A}$.

Let $L$ be the Laurent polynomial associated to the loop around the boundary. Its band graph is the first graph in the last row in Figure 3 .

Lemma 8. $L \in \mathcal{A}_{\text {. }}$.

Proof. Let $V_{1}$ be the cluster variable obtained from $\mathbf{x}$ by the mutation sequence $8,9,10,2,1,9,4,6,3$, and let $V_{2}$ be the cluster variable obtained from $\mathbf{x}$ by the mutation sequence $7,6,5,1,2,6,3,9,4$. Then $V_{1}$ is the cluster variable corresponding to the arc that crosses $8,9,10,2,1,10,4,6,3,8$ and $V_{2}$ is the cluster variable corresponding to the arc that crosses $7,4,9,3,5,2,1,5,6,7$. The corresponding snake graphs $\mathcal{G}_{1}$ and $\mathcal{G}_{2}$ are illustrated on the left-hand side of the equation in Figure 3 ,

Let $X_{i}$ be the Laurent polynomial defined by the band graph obtained from $\mathcal{G}_{i}$, for $i=1,2$, by deleting the first and the last tile and then glueing. These band graphs are illustrated in the last row of Figure 3.

A simple computation using snake graph calculus shows that

$$
V_{1} V_{2}=L+y_{7}\left(y_{8} X_{1}+x_{7}\right)\left(X_{2}+y_{1} y_{2} y_{3} y_{4} y_{5}^{2} y_{6} y_{7} y_{9} x_{8}\right) .
$$

This computation is given in Figure 3. The first equation is the resolution of a grafting, and the second is the resolution of three self-graftings.

In order to show that $L \in \mathcal{A}_{\bullet}$, it suffices to show that $X_{1}$ and $X_{2}$ are in the cluster algebra and, by symmetry, it is enough to show that $X_{1}$ is.

Let $U_{1}$ and $U_{2}$ be the Laurent polynomials associated to the snake graphs on the left-hand side of the equation in Figure 4. It follows from CS2 that none of these two snake graphs has a self-crossing, which implies that $U_{1}$ and $U_{2}$ are cluster 

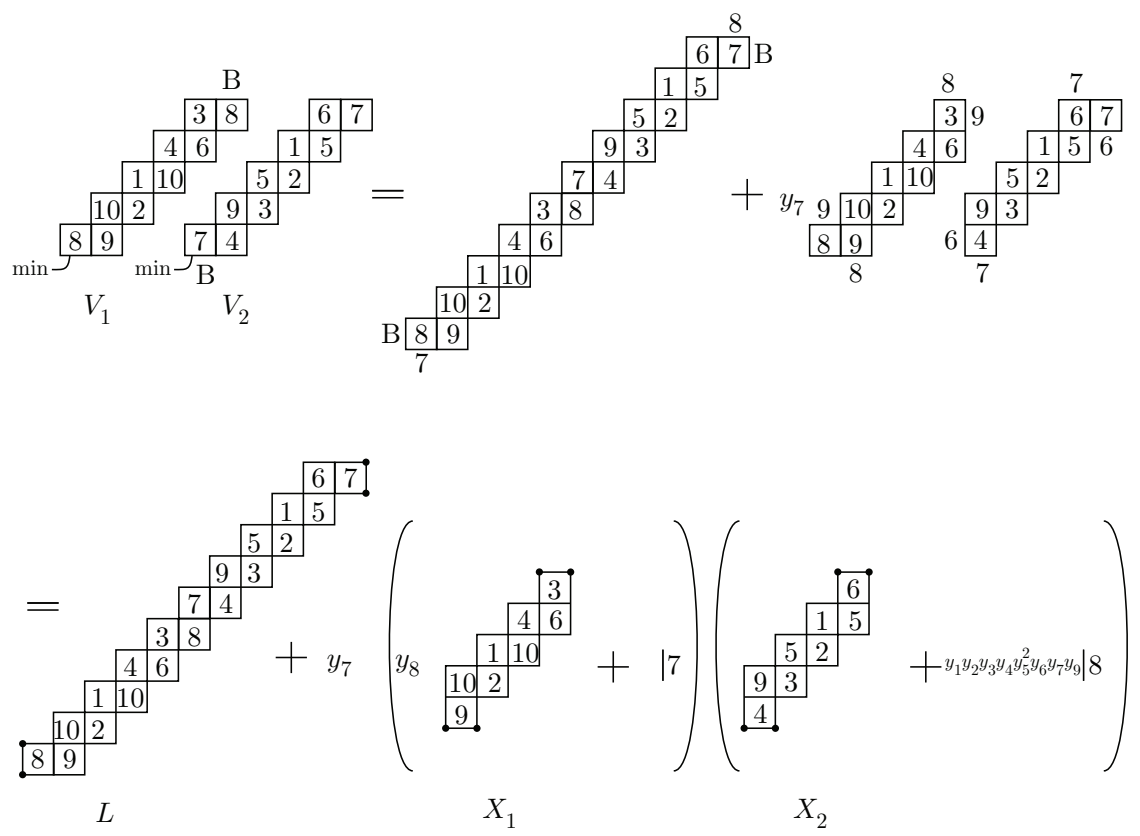

FiguRE 3. Snake graph calculus used in the proof of Lemma 8.

variables. Alternatively, we can see that $U_{1}$ and $U_{2}$ are cluster variables since each corresponds to an arc in the surface; see Figure [5. Indeed, the variable $U_{1}$ corresponds to the arc starting from the marked point into the triangle with sides $3,9,8$, then crossing in order the arcs $3,6,4,10,1,5,6,7$ and ending at the marked point coming from the triangle with sides $B, 8,7$, where $B$ denotes the boundary segment; and the variable $U_{2}$ corresponds to the arc starting from the marked point into the triangle with sides $B, 8,7$, then crossing in order the arcs $8,9,10,2$ and ending at the marked point coming from the triangle with sides $1,2,5$. Since these two curves do not have self-crossings, they are indeed arcs, and hence $U_{1}$ and $U_{2}$ are cluster variables.

Another simple calculation using snake graph calculus shows that

$$
U_{1} U_{2}=y_{1} W_{1}+x_{7}+y_{8} X_{1}+y_{1} y_{8} W_{2}+y_{1} y_{5} y_{6} y_{7} W_{3} x_{1},
$$

where the $W_{i}$ are cluster variables, and their corresponding arcs are illustrated in Figure 5. The computation is shown in Figure 4. The first equality in that figure is obtained by the grafting operation [CS2, section 2.9, case 2], the second equation by the self-grafting operation [CS2, section 3.4], and in the last equation the first two terms are obtained by grafting with a single edge [CS2, section 3.3, case 3] and the third and fourth term by self-grafting.

This shows that $X_{1} \in \mathcal{A}_{\bullet}$ and hence that $L \in \mathcal{A}_{\bullet}$.

Lemma 9. $L \in \hat{\mathcal{A}}$.

Proof. The proof is exactly the same as in the genus 1 case. 


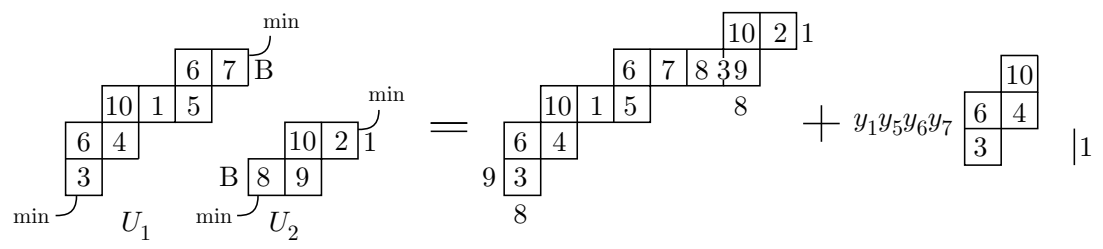

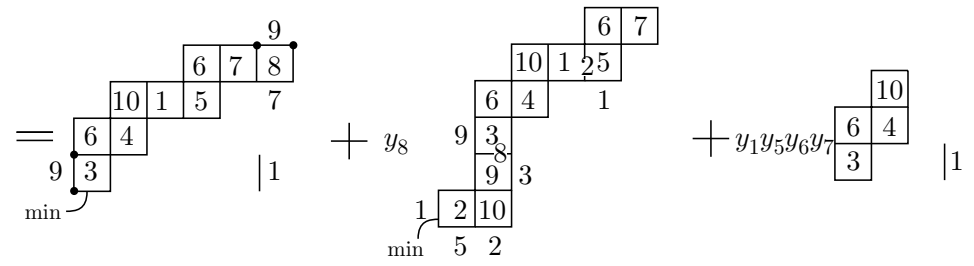

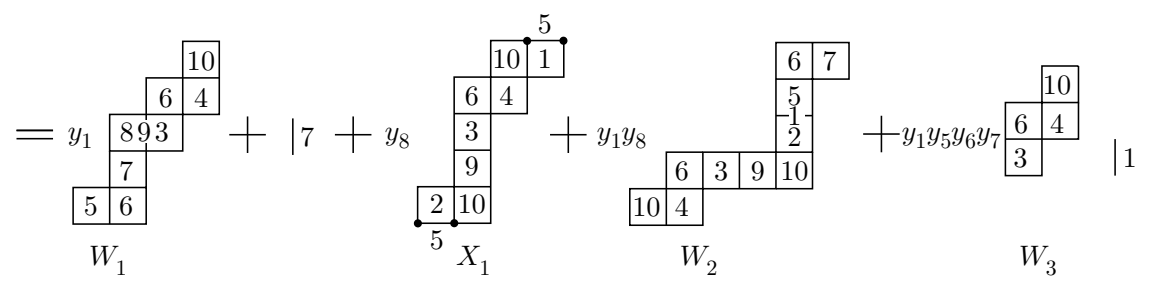

Figure 4. Snake graph calculus showing that

$$
U_{1} U_{2}=y_{1} W_{1}+x_{7}+y_{8} X_{1}+y_{1} y_{8} W_{2}+y_{1} y_{5} y_{6} y_{7} W_{3} x_{1} .
$$
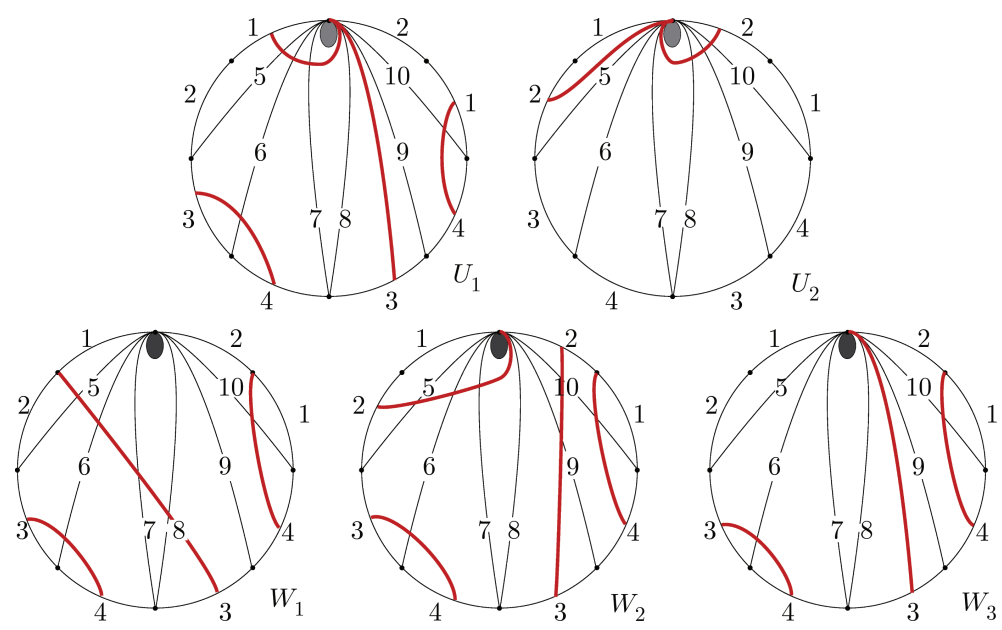

FiguRE 5. The arcs of the cluster variables involved in the computations in genus 2 . 


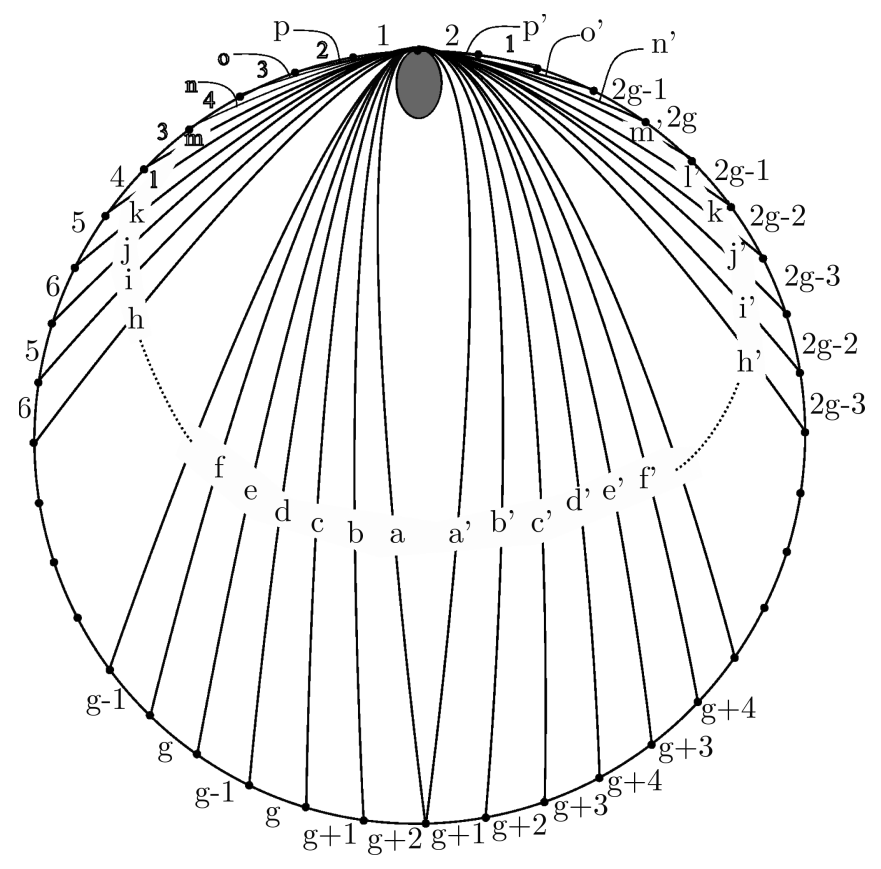

FiguRE 6. Triangulation of a surface of genus $g$ with one boundary component and one marked point.

3.3. Higher genus. In this section, let $(S, M)$ be a surface of genus $g>2$ with one boundary component and one marked point. Fix the triangulation $T$ shown in Figure 6, and let $\mathbf{x}_{T}=\left(x_{1}, \ldots, x_{6 g-2}\right)$ be the corresponding cluster in $\mathcal{A}_{\text {•. Note }}$ that the figure is for $g$ an even integer, but the case where $g$ is odd is similar; only the labeling changes slightly.

As in the genus 1 and 2 cases, let $L$ be the Laurent polynomial associated to the loop around the boundary.

\section{Lemma 10. $L \in \mathcal{A}_{\text {. }}$.}

Proof. The proof is an adaptation of the genus 2 argument. The cluster variables $V_{1}$ and $V_{2}$ are now given by the zigzag snake graphs shown in Figure 7 . The Laurent polynomials $X_{1}, X_{2}$ are again given by the band graphs obtained from the snake graphs of $V_{1}$ and $V_{2}$ by deleting the first and the last tile and then glueing. Again snake graph calculus shows that

$$
V_{1} V_{2}=L+y_{a}\left(y_{a^{\prime}} X_{1}+x_{a}\right)\left(X_{2}+Y x_{a^{\prime}}\right),
$$

where $Y$ is a monomial in $\mathbf{y}$.

Finally to show that $X_{1}$, and hence $X_{2}$, is in the cluster algebra, we use the cluster variables $U_{1}, U_{2}, W_{1}, W_{2}, W_{3}$ analogous to the genus 2 case to get

$$
U_{1} U_{2}=y_{1} W_{1}+x_{a}+y_{a^{\prime}} X_{1}+y_{1} y_{a^{\prime}} W_{2}+y_{1} y_{p} y_{o} \cdots y_{b} y_{a} W_{3} x_{1} .
$$

This shows that $X_{1} \in \mathcal{A}$ and we are done.

Lemma 11. $L \in \hat{\mathcal{A}}$.

Proof. The proof is exactly the same as in the genus 1 case. 


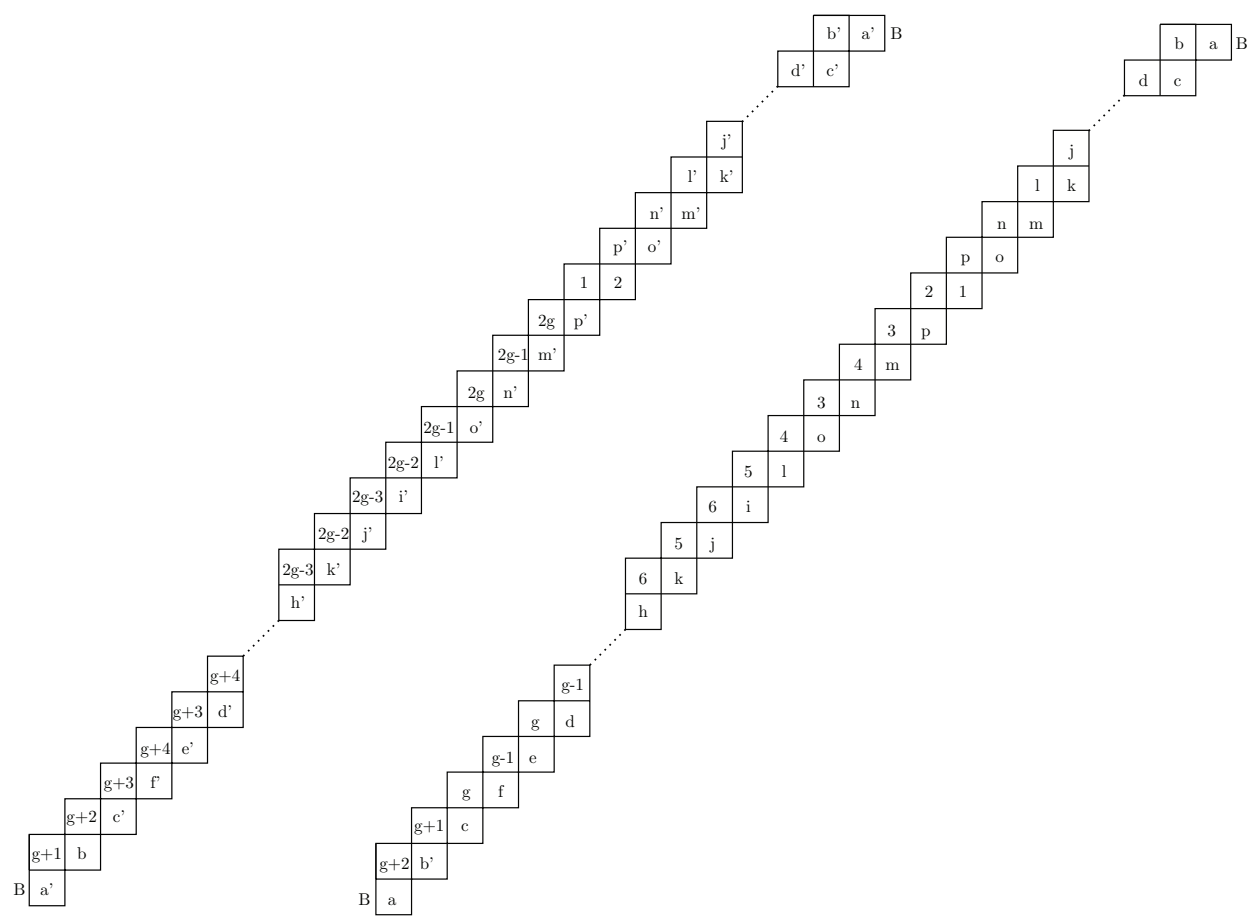

FiguRE 7. Snake graphs of the cluster variables $V_{1}$ (left) and $V_{2}$ (right).

\section{Proof of Lemma 4}

Let $\zeta$ be an essential loop and let $x_{\zeta}$ be the Laurent polynomial associated to it. In view of Lemma 3 we may suppose that $\zeta$ is not the loop around the boundary. The proof uses a geometric argument with skein relations, and the relevant curves are illustrated in Figure 8. Choose a point $z$ on $\zeta$ and a simple curve $\alpha$ that goes from the marked point to the point $z$. Let $\gamma$ be the arc obtained by the curve $\alpha \zeta \alpha^{-1}$. Let $\delta$ be the generalized arc that starts at the marked point, goes around the boundary twice and then ends at the marked point. The curves $\alpha, \zeta, \delta$ and $\gamma$ are illustrated in the first row of Figure 8. Let $x_{\alpha}, x_{\zeta}, x_{\delta}$ and $x_{\gamma}$ be the corresponding Laurent polynomials.

We compute the product $x_{\delta} x_{\gamma}$ using skein relations. Thus each equation is obtained by smoothing a crossing of the curves. This computation is illustrated in the second and third row of Figure 8 . The first term on the right-hand side of the equation in the second row is a cluster variable $x_{\epsilon}$ given by the blue arc $\epsilon$ (the red arc is the boundary segment $B$ ) and the second term on the right-hand side still has a self-crossing. Applying the skein relations to that self-crossing produces the third row in the figure. In this row all red and green curves are boundary segments. Thus we get

$$
x_{\delta} x_{\gamma}=Y_{1} x_{\epsilon}+Y_{2} x_{\zeta}+Y_{3} x_{\sigma},
$$

where $Y_{i}$ are some monomials in $\mathbf{y}$ and $x_{\gamma}, x_{\epsilon}$ and $x_{\sigma}$ are cluster variables. Thus in order to show that $x_{\zeta} \in \mathcal{A}$, it suffices to show that $x_{\delta} \in \mathcal{A}$. But it is shown in the 


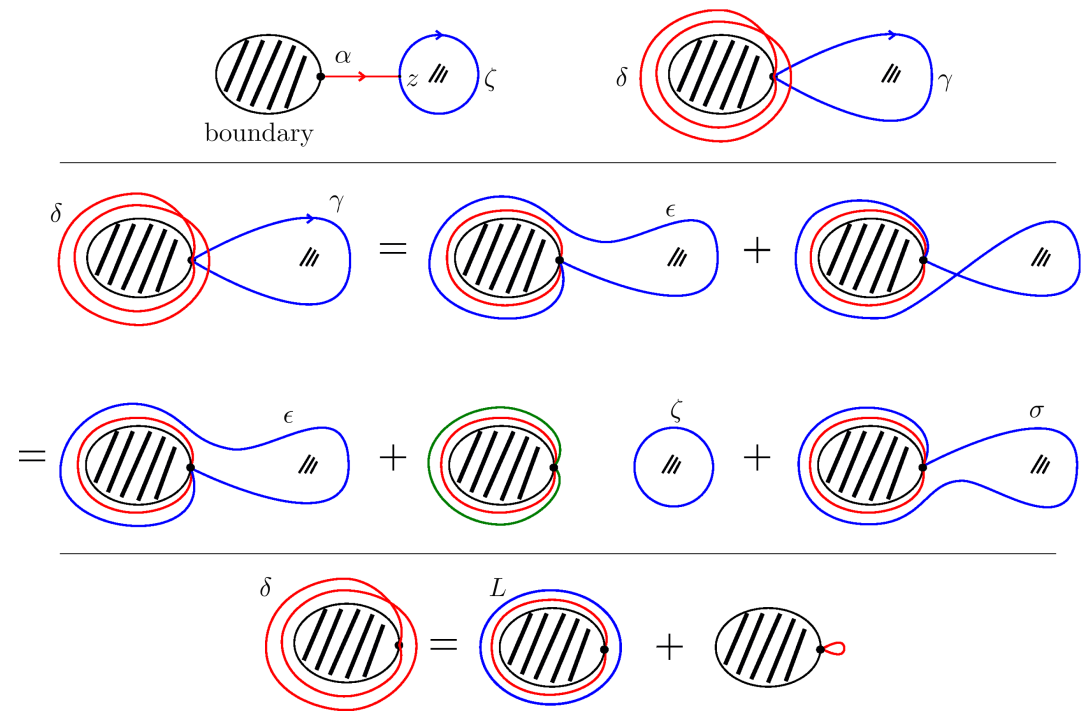

Figure 8. Proof of Lemma 4.

last row of Figure 8 that $x_{\delta}=L+0$, where $L$ (in blue) is the essential loop around the boundary. Now the result follows from Lemma 3 .

\section{Punctured Surfaces With Boundary}

In this section, we show how to reduce the case of punctured surfaces with exactly one marked point on the boundary to the case of surfaces with two marked points on the boundary.

Let $(S, M)$ be a punctured surface with boundary and one marked point on the boundary. Let $\mathcal{A}$ be its cluster algebra and $\mathcal{U}$ the corresponding upper cluster algebra.

Proposition 12. $\mathcal{A}=\mathcal{U}$.

Proof. By $\mathrm{MSp}$ it suffices to show that there is a triangulation whose quiver satisfies the Louise property. Let $p_{0}$ be the marked point on the boundary and let $p_{1}$ be a puncture. Let $\alpha, \beta$ be two arcs going from $p_{0}$ to $p_{1}$ and let $\gamma$ be the arc from $p_{0}$ to $p_{0}$ going around the puncture $p_{1}$ such that $\gamma$ cuts the surface into two pieces, one of which contains the boundary, the puncture $p_{1}$ and no other punctures; see the picture on the left-hand side of Figure 9. If the genus of $S$ is zero and $p_{1}$ is the only puncture, then the cluster algebra is of type $\mathbb{A}_{1}$ and $\mathcal{A}=\mathcal{U}$.

Otherwise, $\gamma$ is not contractible, hence an arc, and we can choose a triangulation $T$ containing the $\operatorname{arcs} \alpha, \beta, \gamma$. Then in the corresponding quiver $Q_{T}$ the vertices $\alpha$ and $\beta$ are sinks or sources. Now the quiver obtained by removing the vertices $\alpha$ and $\beta$ from the quiver $Q_{T}$ is the same as the quiver $Q_{T^{\prime}}$ of the triangulation $T^{\prime}=T-\{\alpha, \beta\}$ obtained by deleting the $\operatorname{arcs} \alpha$ and $\beta$ of the surface $\left(S^{\prime}, M^{\prime}\right)$ obtained by replacing the puncture $p_{1}$ by a second point on the boundary; see the picture on the right-hand side of Figure 9. By induction, $Q_{T^{\prime}}$ is Louise since $\left(S^{\prime}, M^{\prime}\right)$ has two boundary points and one puncture less than $(S, M)$. 


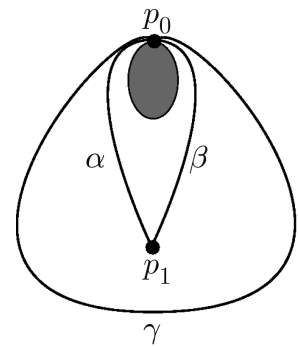

$(S, M)$

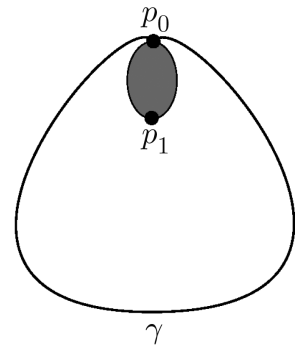

$\left(S^{\prime}, M^{\prime}\right)$

Figure 9. Proof of Proposition 12.

\section{ACKNOWLEDGEMEnts}

Part of this work was carried out at the Centre de Recherche Mathématiques in Montréal, and the authors thank the CRM for their hospitality. The authors also thank Greg Muller for stimulating discussions.

\section{REFERENCES}

[ACCERV] Murad Alim, Sergio Cecotti, Clay Córdova, Sam Espahbodi, Ashwin Rastogi, and Cumrun Vafa, BPS quivers and spectra of complete $\mathcal{N}=2$ quantum field theories, Comm. Math. Phys. 323 (2013), no. 3, 1185-1227, DOI 10.1007/s00220-013-1789-8. MR.3106506

[BDP] Thomas Brüstle, Grégoire Dupont, and Matthieu Pérotin, On maximal green sequences, Int. Math. Res. Not. IMRN 16 (2014), 4547-4586. MR3250044

[BFZ] Arkady Berenstein, Sergey Fomin, and Andrei Zelevinsky, Cluster algebras. III. Upper bounds and double Bruhat cells, Duke Math. J. 126 (2005), no. 1, 1-52, DOI 10.1215/S0012-7094-04-12611-9. MR2110627 (2005i:16065)

[CS] Ilke Canakci and Ralf Schiffler, Snake graph calculus and cluster algebras from surfaces, J. Algebra 382 (2013), 240-281, DOI 10.1016/j.jalgebra.2013.02.018. MR.3034481

[CS2] Ilke Canakci and Ralf Schiffler, Snake graph calculus and cluster algebras from surfaces II: Self-crossing snake graphs, Math. Z. 281 (2015), no. 1-2, 55-102, DOI 10.1007/s00209-015-1475-y. MR3384863

[CS3] Ilke Canakci and Ralf Schiffler, Snake graph calculus and cluster algebras from surfaces III, preprint, arxiv:1506.01742.

[FeShTu] Anna Felikson, Michael Shapiro, and Pavel Tumarkin, Skew-symmetric cluster algebras of finite mutation type, J. Eur. Math. Soc. (JEMS) 14 (2012), no. 4, 1135-1180, DOI 10.4171/JEMS/329. MR2928847

[FeShTu2] Anna Felikson, Michael Shapiro, and Pavel Tumarkin, Cluster algebras of finite mutation type via unfoldings, Int. Math. Res. Not. IMRN 8 (2012), 1768-1804, DOI 10.1093/imrn/rnr072. MR2920830

[FeShTu3] Anna Felikson, Michael Shapiro, and Pavel Tumarkin, Cluster algebras and triangulated orbifolds, Adv. Math. 231 (2012), no. 5, 2953-3002, DOI 10.1016/j.aim.2012.07.032. MR2970470

[GLS] C. Geiss, D. Labardini-Fragoso, and J. Schröer, The representation type of Jacobian algebras, arXiv: 1308.0478.

[FG1] Vladimir Fock and Alexander Goncharov, Moduli spaces of local systems and higher Teichmüller theory, Publ. Math. Inst. Hautes Études Sci. 103 (2006), 1-211, DOI 10.1007/s10240-006-0039-4. MR2233852 (2009k:32011)

[FG2] Vladimir V. Fock and Alexander B. Goncharov, Cluster ensembles, quantization and the dilogarithm (English, with English and French summaries), Ann. Sci. Éc. Norm. 
Supér. (4) 42 (2009), no. 6, 865-930, DOI 10.1007/978-0-8176-4745-2_15. MR.2567745 (2011f:53202)

[FST] Sergey Fomin, Michael Shapiro, and Dylan Thurston, Cluster algebras and triangulated surfaces. I. Cluster complexes, Acta Math. 201 (2008), no. 1, 83-146, DOI 10.1007/s11511-008-0030-7. MR 2448067 (2010b:57032)

[FT] Sergey Fomin and Dylan Thurston, Cluster algebras and triangulated surfaces. Part II: Lambda lengths, preprint (2008), http://www.math.1sa.umich.edu/ $\sim$ fomin/Papers/cats2.ps

[FZ1] Sergey Fomin and Andrei Zelevinsky, Cluster algebras. I. Foundations, J. Amer. Math. Soc. 15 (2002), no. 2, 497-529 (electronic), DOI 10.1090/S0894-0347-01-00385-X. MR:1887642 (2003f:16050)

[FZ2] Sergey Fomin and Andrei Zelevinsky, Cluster algebras. II. Finite type classification, Invent. Math. 154 (2003), no. 1, 63-121, DOI 10.1007/s00222-003-0302-y. MR2004457 (2004m:17011)

[FZ4] Sergey Fomin and Andrei Zelevinsky, Cluster algebras. IV. Coefficients, Compos. Math. 143 (2007), no. 1, 112-164, DOI 10.1112/S0010437X06002521. MR2295199 (2008d:16049)

[GSV] Michael Gekhtman, Michael Shapiro, and Alek Vainshtein, Cluster algebras and WeilPetersson forms, Duke Math. J. 127 (2005), no. 2, 291-311, DOI 10.1215/S0012-709404-12723-X. MR.2130414 (2006d:53103)

[GY] Kenneth R. Goodearl and Milen T. Yakimov, Quantum cluster algebras and quantum nilpotent algebras, Proc. Natl. Acad. Sci. USA 111 (2014), no. 27, 9696-9703, DOI 10.1073/pnas.1313071111. MR3263301

[K] Bernhard Keller, On cluster theory and quantum dilogarithm identities, Representations of algebras and related topics, EMS Ser. Congr. Rep., Eur. Math. Soc., Zürich, 2011, pp. 85-116, DOI 10.4171/101-1/3. MR 2931896

[Lad] S. Ladkani, On cluster algebras from once punctured closed surfaces, preprint, arXiv: 1310.4454.

[LS] Kyungyong Lee and Ralf Schiffler, Positivity for cluster algebras, Ann. of Math. (2) 182 (2015), no. 1, 73-125, DOI 10.4007/annals.2015.182.1.2. MR3374957

[L1] George Lusztig, Canonical bases arising from quantized enveloping algebras, J. Amer. Math. Soc. 3 (1990), no. 2, 447-498, DOI 10.2307/1990961. MR.1035415|(90m:17023)

[L2] George Lusztig, Introduction to quantum groups, Progress in Mathematics, vol. 110, Birkhäuser Boston, Inc., Boston, MA, 1993. MR1227098 (94m:17016)

[MM] J. Matherne and G. Muller, Computing upper cluster algebras, to appear in Int. Math. Res. Not., arxiv:1307.0579.

[M] Greg Muller, Skein algebras and cluster algebras of marked surfaces, preprint, arXiv: 1204.0020.

[M2] Greg Muller, Locally acyclic cluster algebras, Adv. Math. 233 (2013), 207-247, DOI 10.1016/j.aim.2012.10.002. MR.2995670

[M3] Greg Muller, $\mathcal{A}=\mathcal{U}$ for locally acyclic cluster algebras, SIGMA Symmetry Integrability Geom. Methods Appl. 10 (2014), Paper 094, 8, DOI 10.3842/SIGMA.2014.094. MR 3261850

[M4] Greg Muller, private communication.

[MSp] Greg Muller and D. Speyer, Cluster algebras of grassmannians are locally acyclic, preprint, arXiv: 1401.5137.

[MS] Gregg Musiker and Ralf Schiffler, Cluster expansion formulas and perfect matchings, J. Algebraic Combin. 32 (2010), no. 2, 187-209, DOI 10.1007/s10801-009-0210-3. MR:2661414 (2011m:13047)

[MSW] Gregg Musiker, Ralf Schiffler, and Lauren Williams, Positivity for cluster algebras from surfaces, Adv. Math. 227 (2011), no. 6, 2241-2308, DOI 10.1016/j.aim.2011.04.018. MR.2807089 (2012f:13052)

[MSW2] Gregg Musiker, Ralf Schiffler, and Lauren Williams, Bases for cluster algebras from surfaces, Compos. Math. 149 (2013), no. 2, 217-263, DOI 10.1112/S0010437X12000450. MR3020308

[MW] Gregg Musiker and Lauren Williams, Matrix formulae and skein relations for cluster algebras from surfaces, Int. Math. Res. Not. IMRN 13 (2013), 2891-2944. MR3072996 
[S2] Ralf Schiffler, A cluster expansion formula ( $A_{n}$ case), Electron. J. Combin. 15 (2008), no. 1, Research paper 64, 9. MR2398856 (2009d:13029)

[S3] Ralf Schiffler, On cluster algebras arising from unpunctured surfaces. II, Adv. Math. 223 (2010), no. 6, 1885-1923, DOI 10.1016/j.aim.2009.10.015. MR2601004 (2011c:13048)

[ST] Ralf Schiffler and Hugh Thomas, On cluster algebras arising from unpunctured surfaces, Int. Math. Res. Not. IMRN 17 (2009), 3160-3189, DOI 10.1093/imrn/rnp047. MR 2534994 (2010h:13040)

[Se] Ahmet I. Seven, Maximal green sequences of exceptional finite mutation type quivers, SIGMA Symmetry Integrability Geom. Methods Appl. 10 (2014), Paper 089, 5, DOI 10.3842/SIGMA.2014.089. MR3261855

[T] Dylan Paul Thurston, Positive basis for surface skein algebras, Proc. Natl. Acad. Sci. USA 111 (2014), no. 27, 9725-9732, DOI 10.1073/pnas.1313070111. MR3263305

Department of Mathematics, University of Leicester, University Road, Leicester LE1 7RH, United Kingdom

Current address: Department of Mathematical Sciences, Durham University, Lower Mountjoy, Stockton Road, Durham DH1 3LE, United Kingdom

E-mail address: ilke.canakci@durham.ac.uk

Department of Mathematics, Wayne State University, Detroit, Michigan 48202 and - Center for Mathematical Challenges, Korea Institute for Advanced Study, 85 Hoegiro, Dongdaemun-gu, Seoul 130-722, Republic of Korea

Current address: Department of Mathematics, University of Nebraska-Lincoln, 210 Avery Hall, Lincoln, NE 68588-0130, USA

E-mail address: klee24@unl.edu

E-mail address: klee1@kias.re.kr

Department of Mathematics, University of Connecticut, Storrs, Connecticut 062693009

E-mail address: schiffler@math.uconn.edu 